\title{
Особенности определения качественных свойств Требования к лабораториям
}

\author{
И. В. Болдырев', Т. Я. Селиванова²
}

УДК 543

В 2017 году была принята новая редакция международного стандарта, который был переведен на русский язык и вступил в силу в 2019 году - ГOCT ISO / IEC 17025-2019 Оценка соответствия. Общие требования к компетентности испытательных и калибровочных лабораторий. Некоторые положения документа требуют дополнительных разъяснений. Обсуждаются вопросы определения качественных свойств объекта, которые не выражаются в единицах измерений. Перечислены и подробно рассмотрены примеры качественных свойств, которые могут быть определены. Отмечено, что не разработана общая методология определения качественных свойств, поэтому отдельные удачные попытки установить единый подход к методикам определения отдельных групп качественных свойств не решают проблему в целом. В результате в стандартах можно встретить описания методик, которые очевидно нельзя признать достаточно полными. Приведены рекомендации по верификации методик определения качественных свойств в испытательных лабораториях.

Ключевые слова: испытание, оценка соответствия, стандартные образцы, качественные свойства, верификация, скрининг

\section{Требования к испытанию}

Международный стандарт дает следующее определение термину испытание (testing): "Определение одной или более характеристик объекта оценки соответствия согласно процедуре» [1]. Под процедурой испытания следует понимать методику его проведения, как это указано в п. 7.2.1.1 ГОСТ ISO/IEC 17025 [2]. Характеристика объекта включает информацию, отражающую его количественные и качественные свойства [3]. Новая редакция ISO 17000:2020 [4] содержит примечание к приведенному выше определению испытания, в котором разъясняется, что "результаты испытаний могут быть выражены в конкретных единицах измерений или в виде объективного сравнения с общепринятыми эталонами". Таким образом, из определения вытекает, что

\footnotetext{
ААЦ "Аналитика", Москва, boldyrev@aac-analitica.ru.
}

ФГУП «ВНиим им. Д. И. Менделеева», Санкт-Петербург. в процессе испытаний лаборатории определяют, как количественные, так и качественные свойства. Количественные свойства определяются в процессе измерений, требования к методикам измерений хорошо разработаны и описаны во множестве документов, например в [5-8]. Качественные же свойства (свойство явления, тела или вещества, которое не может быть выражено количественно) измерены быть не могут, но тем не менее их определение по ряду критериев возможно [9]. К качественным свойствам могут быть отнесены:

- цвет краски, запах молока, аромат вина и другие органолептические характеристики;

- характеристики объекта, отражающие способность сохранять присущие свойства после определенных воздействий или известным образом реагировать на них. Например, вибростойкость, ударная прочность, коррозионная стойкость, бензостойкость, термостойкость, коллоидная 
стабильность, растворимость и т. п. Результат определения таких качественных характеристик обычно выражают в форме: выдерживает/не выдерживает или с использованием условной классификации (класс 1Б, слаборастворимый и т. п.);

- подтверждение присутствия (отсутствия) компонента в объекте испытаний, если количественная оценка его содержания не проводится. Например, выявление вируса лейкоза КРС методом ПЦР; определение наличия воды в масле методом нагревания, выявление запрещенных допинговых веществ в моче спортсмена, выявление аллергенов в пищевых продуктах и т.п. Результат определения таких качественных свойств выражается в форме: выявлено/не выявлено присутствие компонента; идентификация наличия / отсутствия ингредиента (минералогический анализ, микробиологический анализ и т.п.). Концентрация вещества не может рассматриваться как качественное свойство, так как она может быть измерена, но в отдельных случаях методики химического анализа помогают установить, например, что определенный параметр ниже или выше установленного значения. Тогда эти результаты могут быть рассмотрены как качественное свойство;

- идентификация объекта по совокупности признаков (судебная экспертиза, выявление ДНК целевых организмов, выявление карантинных объектов, таможенная экспертиза и т. п.). Результат определения таких качественных свойств выражается в форме: соответствует / не соответствует образцу;

- описание структуры. Результат определения таких качественных свойств выражается в форме словесного описания или присвоения значения по установленной шкале: макроструктура стали, стратиграфическое описание, описание дефектов древесины и т. п.;

- выявление дефектов. Результат определения таких качественных свойств выражается в форме: обнаружено/не обнаружено с описанием дефекта и его локализации в случае обнаружения (методы неразрушающего контроля);

- оценки свойства по условным (балльным) шкалам (цветность, интенсивность запаха и вкуса, адгезия лакокрасочных покрытий методом решетчатых надрезов, коррозионная активность топлива и т.п.). Результат определения таких качественных свойств выражается в форме: наименование свойства соответствует определенному показателю согласно градации условной шкалы. Например, адгезия лакокрасочного покрытия соответствует двум баллам по таблице 1 ГОСТ 15140.

Как видно из перечисления, определение качественных свойств широко применяется в самых различных прикладных областях лабораторной деятельности. Требования к качественным свойствам устанавливаются в стандартах и технических условиях на продукцию. Методики определения качественных свойств представлены в отдельных документах, а также входят в состав рекомендаций, описывающих методы испытаний продукции. Но! Общие требования к методикам определения качественных свойств отсутствуют. Отдельные удачные попытки установить единый подход к методикам определения отдельных групп качественных свойств $[10,11,12]$ не решают проблему в целом, так как не разработана общая методология определения качественных свойств. В результате в стандартах можно встретить описания методик, которые очевидно нельзя признать достаточно полными. Примеры приведены в табл. 1.

Таблица 1. Примеры недостаточно полных методик определения качественных свойств

\begin{tabular}{|c|c|c|}
\hline Шифр стандарта & $\begin{array}{l}\text { Определяемая } \\
\text { характеристика }\end{array}$ & Методика \\
\hline $\begin{array}{l}\text { ГОСт 10967-2019 Зерно. } \\
\text { Методы определения запаха } \\
\text { и цвета }\end{array}$ & Цвет & $\begin{array}{l}\text { п. 6.4.1. Цвет зерна определяют визуально, сравнивая с описанием этого } \\
\text { признака в стандартах на исследуемую культуру. } \\
\text { При разногласиях цвет определяют при рассеянном дневном свете }\end{array}$ \\
\hline $\begin{array}{l}\text { ГОСТ 32513-2013 Топлива } \\
\text { моторные. Бензин неэти- } \\
\text { лированный. Технические } \\
\text { условия }\end{array}$ & Внешний вид & $\begin{array}{l}\text { 8.2. Внешний вид } \\
\text { Бензин, налитый в стеклянный цилиндр диаметром 40-55 мм, должен } \\
\text { быть прозрачным и не содержать взвешенных и осевших на дно цилиндра } \\
\text { посторонних примесей, в том числе воды }\end{array}$ \\
\hline $\begin{array}{l}\text { ГОСТ Р 51760-2011 Тара по- } \\
\text { требительская полимерная. } \\
\text { Общие технические условия }\end{array}$ & $\begin{array}{l}\text { Стойкость } \\
\text { К горячей воде }\end{array}$ & $\begin{array}{l}\text { 5.2.5.1. Тара должна сохранять внешний вид, цвет и качество декориро- } \\
\text { вания, не должна деформироваться и растрескиваться при погружении } \\
\text { в горячую воду температурой }(70 \pm 5)^{\circ} \mathrm{C}\end{array}$ \\
\hline
\end{tabular}


Для подобных случаев п. 7.2.1.3 ГОСТ ISO/IEC 17025 [2] прямо требует разработки дополнительных уточнений, например, в форме инструкций, чтобы обеспечить единообразное применение методики в лаборатории. Обычно дополнительная регламентация требуется в части подготовки образца к испытанию, воспроизведения условий испытаний, применяемого оборудования, сопоставления испытуемого образца с образцом сравнения, фиксации первичных наблюдений, обработки первичных наблюдений, принятия решения о присвоении значения свойства, оформления результатов, уничтожения, утилизации или передачи образца заказчику.

Пункт 7.2.1.5 ГOCT ISO / IEC 17025 [2] требует от всех лабораторий еще до внедрения в работу провести верификацию методики с целью подтвердить, что лаборатория может надлежащим образом применять выбранную методику, обеспечивая требуемое исполнение. Это требование распространяется на все методики, включая определение качественных свойств.

При верификации методики определения качественных свойств рекомендуется рассматривать как минимум следующие аспекты: наличие требуемых методикой (с учетом внутренних инструкций) ресурсов, действенность процедур обеспечения качества результатов, уровень достоверности результатов определения качественного свойства.

\section{Требования к ресурсам}

Согласно п. 6.1 ГOCT ISO/IEC 17025-2019 [2] лаборатория должна "располагать персоналом, помещениями, оборудованием".

Общие требования к персоналу изложены в п. 6.2 ГOCT ISO / IEC 17025-2019 [2]. Однако следует помнить, что персонал, привлекаемый к определению качественных свойств, часто должен демонстрировать специфические личные свойства, например, способность к цветовым и обонятельным ощущениям. Методики определения качественных свойств часто не содержат требований к компетентности персонала. В таких случаях они должны быть установлены лабораторией самостоятельно, исходя из своего понимания специфики проведения испытаний.

Общие требования к помещениям изложены в П. 6.3 ГОСТ ISO/IEC 17025-2019 [2]. При этом не следует забывать, что на результаты определения качественных свойств, помимо температуры и влажности, могут оказывать влияние факторы, которые для методик измерений обычно не рассматриваются в таком качестве. К ним можно отнести посторонние запахи, спектральный состав освещения, стерильность. Методики определения качественных свойств часто не содержат требований к помещениям и условиям окружающей среды. В таких случаях они должны быть установлены лабораторией самостоятельно, исходя из своего понимания специфики проведения испытаний.

Общие требования к оборудованию изложены в п. 6.4.1 ГОСТ ISO/IEC 17025-2019 [2]. Следует помнить, что стандарт относит к оборудованию "средства измерения, программное обеспечение, эталоны, стандартные образцы, справочные данные, реактивы, расходные материалы или вспомогательные устройства". Этот перечень может быть расширен с учетом других видов оборудования, "которое необходимо для надлежащего осуществления деятельности и которое может повлиять на ее результаты". Исходя из этого, обсуждая особенности методик определения качественных свойств, можно выделить:

- оборудование для подготовки образцов к испытаниям: мельницы, дробилки, устройства для нанесения покрытий, станки, формы, вырубные штампы и т. п.;

- средства измерений для контроля величины испытательного воздействия, включая термометры, динамометры, фотометры, солемеры, рН-метры и т.п.;

- средства измерений для приготовления образцов сравнения: весы, мерная посуда, кондук-

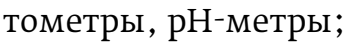

- испытательное оборудование, включая средства воздействия на испытываемый образец: термостаты, печи, криостаты, ударные устройства, грузы, пуансоны, облучатели, магниты и т. п.;

- устройства, приспособления и другие технические средства, предназначенные для закрепления, установки, нагрева, перемешивания, нагружения объектов испытания и(или) испытательного оборудования: цилиндры, колбы, захваты, опоры, штативы, электроплитки, мешалки, подвесы, крепежные скобы и т. п.;

- образцы сравнения, включая стандартные образцы (СО), справочники, коллекции, атласы, испытуемый образец (или его образ) в неизменном состоянии и т. п.;

- растворы установленной концентрации и чистые вещества, воздействующие на образец: дистиллированная вода, солевой раствор, серная кислота, толуол, щелочь, кислород;

- реактивы для приготовления растворов;

- материалы: теплоноситель, рабочее тело, моющие средства, масла, кварцевый песок, фильтры и т. п.;

- программные продукты. 
Прослеживаемость результатов определения качественных свойств имеет две стороны. Первая это прослеживаемость идентичности определения свойства. В измерениях эта проблема решается установлением системы единиц, к которой собственно и обеспечивается прослеживаемость. Для качественных свойств всегда следует помнить, что сначала надо решить, что именно будет определяться и имеются ли в распоряжении лаборатории материальные выражения определяемого свойства в виде образцов сравнения. Вторая - это прослеживаемость результатов измерений величин, воздействующих на объект испытания при определении качественного свойства (температуры, влажности, силы, давления и т. п.). Эта задача является стандартной для любой лаборатории и решается применением калиброванных (поверенных) средств измерения СИ и аттестованного по ГОСТ Р 8.568 испытательного оборудования.

Верификация подразумевает, что лаборатории известны требования к оборудованию, применяемому в ходе проведения испытаний. Если методика не устанавливает этих требований, то в ходе верификации лаборатории следует зафиксировать характеристики использованного оборудования. В дальнейшем, при поступлении новых партий или при замене оборудования, необходимо ориентироваться на эти характеристики при принятии решения о верификации вновь закупаемого оборудования.

Требования к обеспечению достоверности результатов испытаний приведены в п. 7.7 ГOCT ISO/IEC 17025-2019 [2]. Особенностями мониторинга достоверности результатов определения качественных свойств являются:

- практически полное отсутствие СО качественных свойств. В России аттестованное значение СО должно выражаться в допущенных к применению единицах измерения. Поэтому используются либо иностранные СО качественных свойств, либо образцы, которые, по сути, являются $\mathrm{CO}$, но не называются ими (музейные культуры микроорганизмов, мономинеральные фракции, эталонные пластины при определении степени коррозии, образцы из коллекций, эталонные изображения и т. п.);

- использование образцов известного происхождения (с априори известными свойствами) в качестве контрольных, искусственно измененных объектов или объектов с искусственно введенными добавками. Например, молоко, полученное от коровы больной лейкозом КРС; моча человека, принимавшего (не принимавшего) допинговый препарат; образец зерна с внесенным карантинным объектом; проба консервов с добавкой обсемененного материала; образцы с искусственными дефектами и т. п.;

- помещение в единую линейку подвергаемых испытанию и контрольных образцов.

Еще одна особенность методик состоит в том, что, как правило, результат определения качественного свойства неразрывно связан с принятием решения о соответствии (см. п. 7.8.6 ГОСТ ISO/IEC 170252019) [2]. Действительно, если результат испытания характеризует нахождение объекта в одном из двух возможных состояний (выдержал / не выдержал воздействие), то автоматически в первом случае принимается решение о соответствии, а во втором - о несоответствии. Принимая решение о соответствии, необходимо оценить уровень его достоверности (вероятность ложноположительных и ложноотрицательных решений). Ложноположительным является решение о соответствии, принятое в отношении объекта испытания, не соответствующего заданным требованиям. А ложноотрицательным является решение о несоответствии в отношении объекта, отвечающего заданным требованиям. Вероятности ложноположительного и ложноотрицательного решения, как правило, не одинаковы. Более того, попытки уменьшить вероятность ложноположительного решения приводит к увеличению вероятности ложноотрицательного решения. Поэтому правила принятия решений в ходе определения качественного свойства должны "настраивать" на что-то одно: либо выявление соответствия, либо на выявление несоответствия.

Приведем пример. Для контроля качества некоего жидкого продукта установлена методика визуального определения примесей в проходящем свете в столбе жидкости. Если наличие частиц выявляется, то принимается положительное решение. Вероятность ложноположительного решения достаточно низка, так как частицы де-факто обнаружены* . Однако если частицы не обнаружены (отрицательное решение), то вероятность ложноотрицательного решения может быть достаточно высока (частицы не попали в поле зрения, размеры частиц меньше разрешающей способности глаза лаборанта, оптические свойства частицы не позволяют выявить ее в этой жидкости и т.п.). Поэтому для положительного решения результат испытания следует выражать "выявлено наличие частиц", а для отрицательного решения "наличие частиц не выявлено". Выражение в форме «частицы отсутствуют" не должно применяться, так

Даже при обнаружении частиц остается вероятность, что причина их появления связана не с образцом (например, грязная посуда). 
как вероятность ложноотрицательного результата весьма велика.

Особой категорией методик определения качественных свойств является скрининг. Скрининг испытания с целью установления возможного обнаружения компонента. Для этих методик баланс ложноположительности и ложноотрицательности результатов выражен максимально. Низкая вероятность получения ложноотрицательного результата позволяет сделать надежный вывод об отсутствии целевых компонентов, а высокая вероятность ложноположительного результата свидетельствует только о возможном присутствии компонента и часто превышает вероятность получения истинного положительного результата. Примерами скрининга являются методики с применением газосигнализаторов и газоанализаторов с неселективными сенсорами; индикаторных трубок, реагирующих на наличие групп веществ; методики скрининга допинговых и наркотических веществ $[13,14]$. Как правило, при использовании скрининговых методик протокол (отчет) испытаний оформляется только при получении отрицательного результата - "наличие (наименование вещества) не выявлено" или "(наименование компонента) не обнаружен". В противном случае принимают решение о применении другого (подтверждающего) метода для получения количественного результата измерения.

\section{Выводы}

Методикам определения качественных свойств в отечественных стандартах уделяется мало внимания. Эти методики часто недостаточно подробно описаны, что не позволяет единообразно реализовывать их в разных лабораториях.

Из-за неполного описания методик в стандартах лабораториям для их применения требуется разработать дополнительные уточнения, например, в форме инструкций, чтобы обеспечить единообразное применение методики в лаборатории.

Для включения в область аккредитации испытательных лабораторий методик определения качественных свойств необходимо учитывать их особенности.

\section{Литература}

1. ГOCT ISO/IEC 17000-2012 Оценка соответствия. Словарь и общие принципы.

2. ГOCT ISO/IEC 17025-2019 Оценка соответствия. Общие требования к компетентности испытательных и калибровочных лабораторий.

3. ГОСТ Р ИСО 9000-2015 Системы менеджмента качества. Основные положения и словарь.
4. ISO 17000:2020 Conformity assessment. Vocabulary and general principles.

5. ГОСТ 8.563-2009 Государственная система обеспечения единства измерений. Методики (методы) измерений.

6. МИ 1317-2004 Рекомендации по метрологии Государственная система обеспечения единства измерений. Результаты измерений и характеристики погрешности измерений. Формы представления. Способы использования при испытаниях образцов продукции и контроле их параметров.

7. РМГ 61-2010 Рекомендации по межгосударственной стандартизации. Государственная система обеспечения единства измерений. Показатели точности, правильности, прецизионности методик количественного химического анализа. Методы оценки.

8. ГОСТ 34100.3-2017/ISO/IEC Guide 98-3:2008 Неопределенность измерения. Часть 3. Руководство по выражению неопределенности измерения.

9. Руководство ИСО/МЭК 99:2007 (ISO/IEC GUIDE 99:2007) Международный словарь по метрологии. Основные и общие поня тия и соответствующие термины (VIM) (International vocabulary of metrology - Basic and general concepts and associated terms (VIM).

10. ГOCT ISO 6658-2016 Органолептический анализ. Методология. Общее руководство.

11. ГОСТ ISO 7218-2015 Микробиология пищевых продуктов и кормов для животных. Общие требования и рекомендации по микробиологическим исследованиям.

12. ГОСТ 1778-70 Сталь. Металлографические методы определения неметаллических включений.

13. UNODC ST/NAR/45 Руководство по судебной экспертизе наркотиков, с помощью которых совершаются насильственные действия сексуального характера и другие преступные деяния. https://www. unodc.org/documents/scientific/Rape_Drugs_Russian.pdf.

14. UNODC ST/NAR/48 Рекомендуемые методы идентификации и анализа агонистов рецепторов синтетических каннабиноидов в изъятых материалах. https://www.unodc.org/documents/scientific/ Synthetic_Cannabinoids_Manual_Russian.pdf.

\section{References}

1. COST ISO/IEC 17000-2012 Conformity assessment. Vocabulary and general principles.

2. COST ISO/IEC 17025-2019 General requirements for the competence of testing and calibration laboratories.

3. GOST ISO 9001 Quality management systems. Fundamentals and vocabulary.

4. ISO 17000:2020 Conformity assessment. Vocabulary and general principles.

5. COST 8.563 State system for ensuring the uniformity of measurements. Procedures of measurements.

6. MI 1317-2004 Recommendations for metrology. State system for ensuring the uniformity of measurements. Methods of use in testing product samples and monitoring their parameters.

7. RMC 61-2010 Recommendations for interstate standardization. State system for ensuring the uniformity of measurements. Indicators of accuracy, correctness, and precision of quantitative chemical analysis methods. Evaluation methods.

8. GOST 34100.3-2017/ISO/IEC Guide 98-3:2008 Uncertainty of measurement. Part 3. Guide to the expression of uncertainty in measurement.

9. ISO Cuide 99 International vocabulary of metrology - Basic and general concepts and associated terms (VIM).

10. COST ISO 6658-2016 Sensory analysis. Methodology. General guidance.

11. GOST ISO 7218-2015 Microbiology of food and animal feed. General requirements and guidance for microbiological examinations.

12. GOST 1778-70 Steel. Metallographic methods for the determination of nonmetallic inclusions.

13. UNODC ST/NAR/45 Guidelines for the Forensic analysis of drugs facilitating sexual assault and other criminal acts.

14. UNODC ST/NAR/48 Recommended methods for the Identification and Analysis of Synthetic Cannabinoid Receptor Agonists in Seized Materials. 


\section{Системы измерения счетной}

\section{концентрации и размеров частиц}

в маслах, топливах

и фармацевтических препаратах

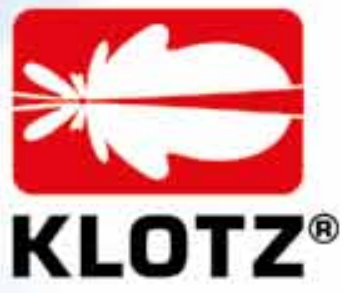

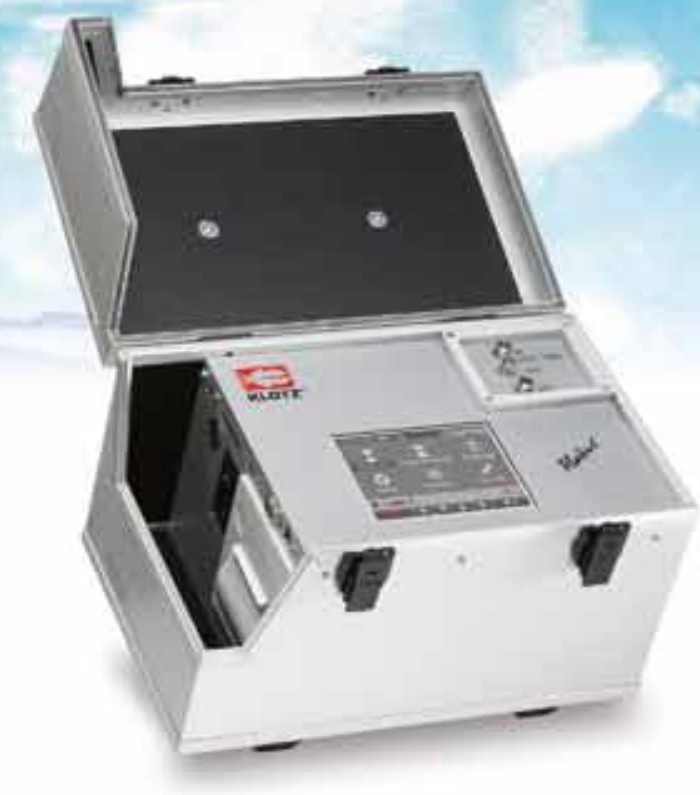

Счетчик частиц в жидкостях SYRINGE ${ }^{\oplus}$

Соответствие фармакопейным статьям Россия: ОФС 1.4.2.0006.15

США: $\quad$ USP $<788>,<787>$, <789>

Европа: $\quad E P<2.9 .19>$

Калибровка

В соответствии C ISO 21501-3-2019

Госреестр СИ №79582-20
Портативный счетчик частиц в жидкостях ABAKUS $^{\circ}$ MOBIL FLUID TOUCH

- ISO 11500, ГOCT 31247-2004

- ASTM D7619, rOCT 3423

- IP 564, IP 565, IP 577, IP PM FA

- ГOCT 17216-2001

- ISO 4406:1987 (1991), ISO 4402:1991

- NAS 1638

- $4,6,10,14,21,25,38,70$ мкм по ISO 4406, SAE 4059

- $2,5,10,15,20,25,50,100$ MKM по ГOCT 17216, ISO 4402, NAS 1638

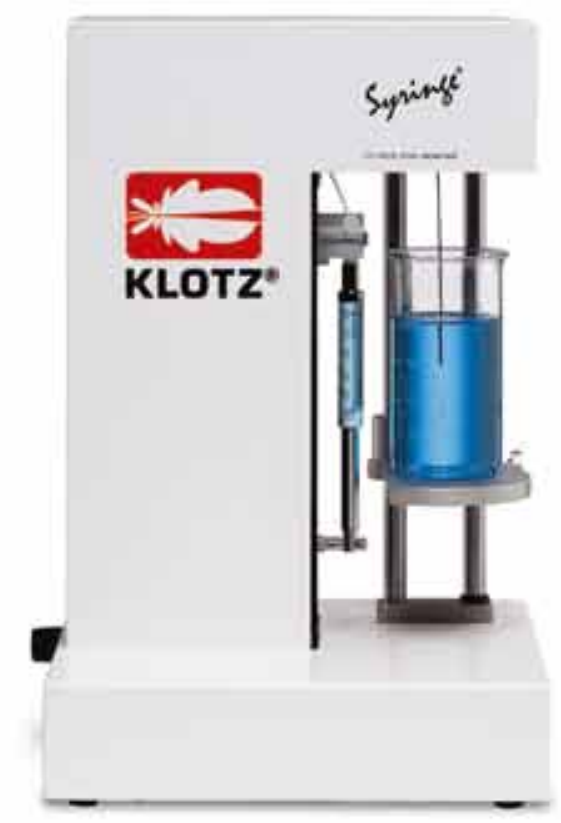

